\title{
Interventions to improve liver enzyme screening testing in obese patients aged $<18$ years in a public hospital, Chicago, IL, 20I7-20I8
}

This article was published in the following Dove Press journal: Pediatric Health, Medicine and Therapeutics

\author{
Megan Ward' \\ Peter Nguyen' \\ Simi Akintorin ${ }^{2}$ \\ Rosibell Arcia' \\ Kenneth Soyemi ${ }^{1,3}$ \\ 'Department of Pediatrics, Cook \\ County Health, and Hospitals System, \\ The John H. Stroger Jr. Hospital, \\ Chicago, IL, USA; ${ }^{2}$ Keck School of \\ Medicine, University of Southern \\ California Medical School, Los \\ Angeles, CA, USA; ${ }^{3}$ Department of \\ Emergency Medicine, Cook County \\ Health, and Hospitals System, The \\ John H. Stroger Jr. Hospital, Chicago, \\ IL, USA
}

Introduction: Our study objective was to determine the health care provider liver enzyme screening testing (LEST) rates in obese pediatric patients at risk for nonalcoholic fatty liver disease (NAFLD), with the goal of improving NAFLD LEST after specific system-wide provider intervention.

Methods: We conducted a bi-phased retrospective electronic medical record review of health care practitioner encounters to determine LEST in overweight/obese (body mass index $\geq 25$ ) patients between ages 2 and 18 years in our outpatient clinics. Intervention activities included lectures to staff and residents, fliers distributed to providers, monthly email reminders, and computer stickers placed on all terminals. From both phases, samples of simple random samples were drawn from the selected electronic medical records and reviewed for LEST screening; after intervention from this pool of patients, a random sample was chosen for LEST rate analysis. LEST rates were calculated per 100 patient encounters.

Results: We screened 2,979 and 2,634 pre and postintervention pediatric encounters from which we obtained a simple random sample of patients for LEST analysis. Overall of the 264 preintervention patients, 65 (24.4\%) patients received LEST translating to 24/100 encounters. Of the 65 who received screening, $53(81 \%)$ were classified as overweight/obese. Screening rate was higher for overweight/obese patients (32/100 encounters), when compared with normal weight patients' crude OR 3.8 ( $11 / 100$ encounters; 95\% CI: 1.9-7.6, $P<0.005)$. Of the 242 postintervention patients, 70 (28\%) received LEST translating to $28 / 100$ encounters; of these, $58(82 \%)$ were classified as overweight/obese with a rate of $42 / 100$ encounters compared with $11 / 100$ encounters for normal weight patients' crude OR 5.5 (95\% CI: $2.8-7.5, P<0.005$ ).

Conclusion: Obesity is a significant risk factor for NAFLD; one in four obese pediatric patients had LEST. Additional health care practitioner education and interventions are needed to improve LEST to prevent long-term complications such as liver cirrhosis or failure.

Keywords: obesity, NAFLD, ALT, screening, pediatric

\section{Introduction}

Obesity is the most significant risk factor associated with nonalcoholic fatty liver disease (NAFLD). The prevalence of NAFLD has increased in parallel with rising trends in overweight and obese pediatric patients in the last three decades. NAFLD prevalence has increased and during the next 10 years is expected to be the leading cause of liver pathology in the Western world; NAFLD may progress to liver failure and consequently the need of hepatic transplant. ${ }^{1,2}$ Prevalence of NAFLD among the general pediatric population ranges from $3 \%$ to $9 \%$ and could be as high as $80 \%$ in obese cohort. ${ }^{3}$

NAFLD screening is important because it can be detected before the onset of irreversible, end-stage liver disease. Identification of NAFLD in children is vital because Email ksoyemi@cookcountyhhs.org in in 
weight reduction and management if initiated early will reduce complications such as advanced liver fibrosis and failure. Our study aim was to determine liver enzyme screening testing (LEST) rates in obese pediatric patients at risk for NAFLD among providers within our hospital system with the goal of improving LEST rates after the system-wide intervention.

\section{Methods}

With the help of information technology group and using body mass index (BMI) parameters, we conducted two phases of electronic medical record (EMR) review of health care practitioner patient encounters to determine LEST rates in patients between ages 2 and 18 years in our outpatient clinics. The preintervention phase EMR review occurred from March through August 2017, and the postintervention phase from September 2017 through February 2018.

\section{Intervention}

To improve LEST rate, in August 2017, we competed a brown bag lunch lecture that addressed natural history of NAFLD and the screening recommendations from North American Society for Pediatric Gastroenterology, Hepatology and Nutrition. ${ }^{4}$ Participants included general pediatricians and pediatric residents providing care in outpatient settings. From September 2017 to February 2018, six monthly screening reminders (verbal and by email) were provided to those in the initial lecture attendance and to all pediatric providers. Email included an attachment of a copy of liver screening algorithm for clinical reference. Subsequently a standardized monthly email including guidelines for initiating screening measures based on BMI, age, and risk factors was sent to providers as promoters or enhancers. Guidance for liver enzyme screening follow-up and management referenced within the full algorithm was attached to the email. During the 6-month intervention period, sticker reminders emphasizing recommended screening (BMI, age, and risk factors) were placed in eight clinical charting areas within the outpatient pediatric clinic to enhance liver enzyme screening. Patients with abnormal liver screen were referred to pediatrics gastroenterology for additional work up according to the protocol.

\section{Statistical analysis}

For both phases, simple random samples (SRSs) were drawn from the pool of EMRs and reviewed for LEST screening. We calculated our SRS by using the sample size for a proportion method. We hypothesized percent frequency of LEST screening in the population of $50 \%$, confidence limits of $95 \%$, design effect of 1 , and power of 0.80 . LEST rates were calculated per
100 patient encounters; qualitative variables were expressed as percentages. Quantitative variables were expressed as mean values $( \pm \mathrm{SD})$ if they followed a normal distribution or as median values (interquartile range $[\mathrm{IQR}]$ ) if otherwise. For bivariate analysis, we used $\mathrm{BMI} \geq 25$ to divide patients into two groups: normal weight and overweight/obese. We calculated the odds of being screened and reported corresponding ORs and their corresponding $95 \% \mathrm{CI}$. We regressed ALT on the BMI to determine the direction and strength of a relationship (if any) and completed model diagnostics by plotting the residuals vs fitted data. Data preparation and analysis were completed using IBM PASW SPSS version 25 ${ }^{\mathrm{TM}}$, Minitab $^{\mathrm{TM}}$, and Microsoft Excel ${ }^{\mathrm{TM}}$.

\section{Ethical considerations}

For ethical consideration, the study was approved by an expedited review by the Cook County Health \& Hospitals System Institutional Review Board (CCHHS IRB) August 8, 2017 study number 17-584. Parental consent to review medical records was not required by the IRB because of the retrospective nature of the study; in addition, data confidentiality followed the principles of the Declaration of Helsinki.

\section{Result}

We screened 2,979 preintervention and 2,634 postintervention pediatric encounters from which we obtained SRS of 264 (pre) and 242 (post) encounters, respectively for the LEST analysis. For study demographics, see Table 1. Overall of the 264 preintervention patients, $65(24.4 \%)$ patients received LEST translating to 24/100 encounters. Of the 65 who received screening, 53 (81\%) were classified as overweight/obese. Screening rate was higher for overweight/obese

Table I Comparison of preintervention with postintervention patient samples

\begin{tabular}{|l|l|l|}
\hline Factor & $\begin{array}{l}\text { Preintervention, } \\
\text { N=264 }\end{array}$ & $\begin{array}{l}\text { Postintervention, } \\
\mathbf{N}=\mathbf{2 4 2}\end{array}$ \\
\hline $\begin{array}{l}\text { Age in years, } \\
\text { Mean (SD) }\end{array}$ & $10.3(4.8)$ & $10.2(4.7)$ \\
BMI, mean (SD) & $27.6(6.8)$ & $26.8(6.5)$ \\
BMI\%, mean (SD) & $98.1(1.4)$ & $97.1(3.1)$ \\
ALT, mean (SD) & $40.4(9.6)$ & $43.4(4 I .2)$ \\
Male, N (\%) & $128(48.4)$ & $122(50.4)$ \\
White, N (\%) & $158(59.8)$ & $165(68.2)$ \\
Hispanic, N (\%) & $145(54.9)$ & $157(65.1)$ \\
Black, N (\%) & $80(33.3)$ & $73(30.3)$ \\
Overweight/ & $162(61)$ & $137(57)$ \\
obese, N (\%) & & $70(28)$ \\
LEST screening, & $65(23)$ & \\
N (\%) & & $5.5(2.8-10.9)$ \\
Crude OR (95\% & $3.8(I .9-7.6)$ & \\
Cl) & & \\
\hline
\end{tabular}

Abbreviations: BMI, body mass index; LEST, liver enzyme screening testing. 
patients (32/100 encounters), when compared with normal weight patients' crude OR 3.8 (11/100 encounters; 95\% CI: 1.9-7.6, $P<0.005)$. Of the 242 postintervention patients, 70 (28\%) received LEST translating to $28 / 100$ encounters; of these, 58(82\%) were classified as overweight/obese with a rate of 42/100 encounters compared with 11/100 encounters for normal weight patients' crude OR 5.5 (95\% CI: 2.8-7.5, $P<0.005)$. With a cutoff screening of ALT $=30$, in the preintervention period $16(24 \%)$ of 65 had an ALT $>30$; of these $14(88 \%)$ were obese compared with $23(33 \%)$ of 70 of which $21(91 \%)$ were obese in the postintervention period.

\section{Discussion}

Demographics of patients sampled in both phases were similar (Table 1); our study demonstrated improvement in LEST, especially in the overweight/obese cohort after system-wide mitigation. Provider participation in intervention activities was high, and as expected we saw bidimensional improvement in both rates and odds of screening among the overweight/obese cohort. In addition, when blood samples were obtained, mean (SD) ALT was higher in obese cohort patients, when compared with the normal weight cohort 40.4 (41.1) vs 19.8 (9.6) $[P<0.01]$ for the preintervention phase and $43.4(41.2)$ vs 20.6
(9.3) $[P<0.01]$ for the postintervention phase. Linear regression analysis of our data showed the statistically significant relationship between ALT and BMI with a regression line of $-18.5+1.65$ $\times(\mathrm{BMI}), R^{2}=0.10$, meaning BMI explained $10 \%$ of the variation in ALT levels. When stratified by study phase, the regression line for preintervention phase for ALT $=-18.60+1.65 \times(\mathrm{BMI})$ with an $R^{2}=0.13$, compared with ALT $=-19.02+1.61 \times(\mathrm{BMI})$ with an $R^{2}=0.08$ for the postintervention phase. For regression diagnostics, we plotted the residuals (the difference between actual and predicted) against the fitted data. The diagnostic plot showed a nonrandom distribution on both sides of zero (Figure 1), emphasizing the relationship between ALT and BMI. In overweight or obese pediatric patients, NAFLD should be suspected in the presence of elevated ALT combined with ultrasonographic findings. Blood samples for ALT in the presence of disease is often two to three times the upper limit of normal. Studies have demonstrated that ALT is neither sensitive nor specific for NAFLD; values fluctuate and may even be normal in patients with simple steatosis, nonalcoholic steatotic hepatitis, or cirrhosis. ALT threshold for the diagnosis of the liver disease varies among clinical sites. ${ }^{4,5}$ Our analysis confirmed a statistically significant relationship between ALT and BMI with low $R^{2}$, meaning that even though NAFLD may be pres-

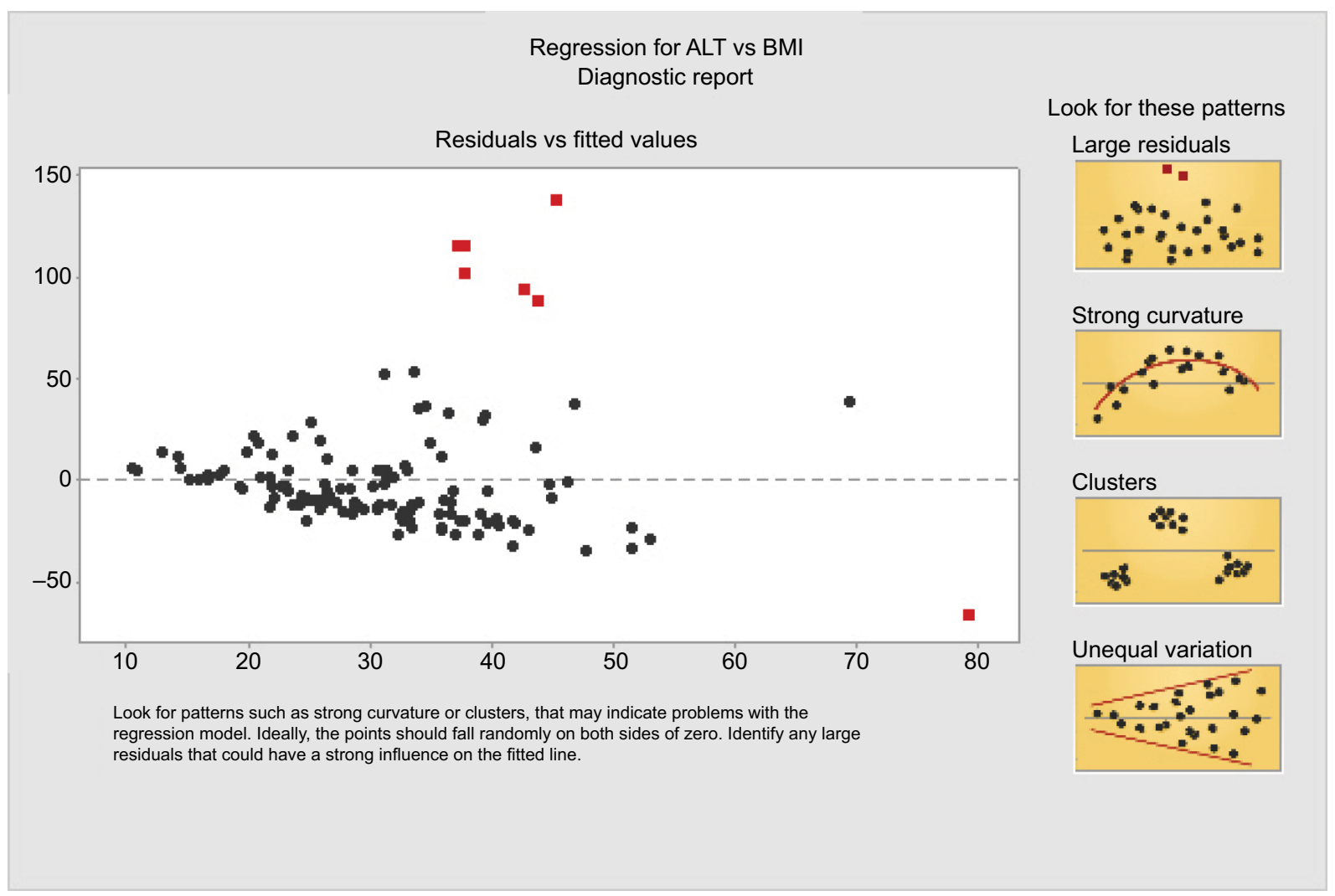

Figure I Scatter plot of residuals vs fitted values of BMI and ALT.

Abbreviation: BMI, body mass index. 
ent, the patient may be asymptomatic until there is significant liver damage. Nonalcoholic steatotic hepatitis is an advanced form of NAFLD, ${ }^{3}$ therefore, it is important for pediatricians to promptly identify and screen at-risk patients to avoid complications such as liver cirrhosis or failure. We demonstrated an improvement in LEST rate after focused mitigation strategy; to augment the screening efforts we recommend early weight loss reduction, optimal ideal body weight maintenance, and nutritionally balanced diet as initial clinical management tools.

\section{Disclosure}

The authors report no conflicts of interest in this work.

\section{References}

1. Satapathy SK, Sanyal AJ. Epidemiology and natural history of nonalcoholic fatty liver disease. Semin Liver Dis. 2015;35(3):221-235.

2. Brunt EM. Nonalcoholic fatty liver disease and the ongoing role of liver biopsy evaluation. Hepatol Commun. 2017;1(5):370-378.

3. Nobili V, Alkhouri N, Alisi A, et al. Nonalcoholic fatty liver disease: a challenge for pediatricians. JAMA Pediatr. 2015;169(2): $170-176$.

4. Vos MB, Abrams SH, Barlow SE, et al. NASPGHAN Clinical Practice Guideline for the diagnosis and treatment of nonalcoholic fatty liver disease in children: recommendations from the Expert Committee on NAFLD (ECON) and the North American Society of Pediatric Gastroenterology, Hepatology and Nutrition (NASPGHAN). J Pediatr Gastroenterol Nutr. 2017;64(2):319-334.

5. Fusillo S, Rudolph B. Nonalcoholic fatty liver disease. Pediatr Rev. 2015;36(5):198-206.
Pediatric Health, Medicine and Therapeutics

\section{Publish your work in this journal}

Pediatric Health, Medicine and Therapeutics is an international, peerreviewed, open access journal publishing original research, reports, editorials, reviews and commentaries. All aspects of health maintenance, preventative measures and disease treatment interventions are addressed within the journal. Practitioners from all disciplines are invited to submit

\section{Dovepress}

their work as well as healthcare researchers and patient support groups The manuscript management system is completely online and includes a very quick and fair peer-review system. Visit http://www.dovepress.com/ testimonials.php to read real quotes from published authors.

Submit your manuscript here: http://www.dovepress.com/pediatric-health-medicine-and-therapeutics-journal 